\title{
Infer the Meaning of Unknown Words by Sheer Guess or by Clues? - An Exploration on the Clue Use in Chinese EFL Learner's Lexical Inferencing
}

\author{
Zhaochun Yin $^{1}$ \\ ${ }^{1}$ School of International Education, Zhengzhou University, Zhengzhou, Henan, China \\ Correspondence: Dr. Zhaochun Yin, Room 101, School of International Education, Zhengzhou University, 100th \\ Science Avenue, Zhengzhou, Henan, 453001, China. Tel: 86-371-6773-9529. E-mail: \\ zhaochunyin@hotmail.com
}

Received: May 31, 2013 Accepted: August 22, 2013 Online Published: October 10, 2013

doi:10.5539/elt.v6n11p29 URL: http://dx.doi.org/10.5539/elt.v6n11p29

\begin{abstract}
Lexical inferencing is refered to as guessing the meaning of an unknown word using available linguistic and other clues. It is a primary lexical processing strategy to tackle unknown words while reading. This study aims to explore the clue use of Chinese EFL learners in inferring the meaning of unknown word in reading. Two types of introspective research methods have been used to achieve the research aim. 55 participants of four levels (tertiary final, tertiary middle, tertiary initial and senior secondary) were asked to read a sample text and infer the meaning of target words through thinking aloud. Additional information of their lexical inferencing was elicited through stimulated recalls. The results show that Chinese EFL learners use a variety of clues in their lexical inferencing. The findings also reveal some discrepancies of clue use across different levels learners in lexical inferencing.
\end{abstract}

Keywords: EFL lexical inferencing, clue use

\section{Introduction}

In reading, lexical inferencing is refered to as guessing the meaning of an unknown word using available linguistic and other clues (Haastrup, 1991). Lexical inferencing is the 'primary lexical processing strategy' (Fraser, 1999, p. 230) that L2 learners rely on when encountering unknown words while reading (de Bot, Paribakht, \& Wesche, 1997; Fraser, 1999; Paribakht, \& Wesche, 1999). Studies also reveal high correlations between lexical inferencing success and reading comprehension, vocabulary knowledge, and reading skill (Herman, Anderson, Pearson, \& Nagy, 1987). Lexical inferencing, in most cases, is the initial stage of incidental acquisition of vocabulary. As a result, investigation in lexical inferencing process may potentially contribute to its success and to subsequent vocabulary acquisition.

The aim of this study is to explore the full view of Chinese EFL learners in lexical inferencing, which includes the clue use, the procedure, the success rate, and the development of vocabulary knowledge after inference. This article only focuses on clue use, and other aspects will be presented separately in other essays.

\section{Literature Review}

The terms "clue" and "cue", used interchangeably in this article, is refered to as information conducive in lexical inferencing. In the past several decades, a number of studies have been carried out to identify and classify various clues of lexical inferencing. Ames (1966) identified fourteen types of clue used by native speakers, such as, comparison/contrast, definition, cause/effect, question/answer, and main idea/details. Carton (1971) classified all types of cues used by L2 readers into three categories: intra-lingual, inter-lingual and extra-lingual cues. In his explanatory framework, Sternberg (1987) claimed that there were eight types of contextual cues (temporal cues, spatial cues, value cues, stative descriptive cues, functional descriptive cues, causal/enablement cues, class membership cues, and equivalence cues). Similar to Carton, Haastrup (1991) also classified clues used by readers into intra-lingual, inter-lingual, and contextual types. Based on their previous research, Paribakht and Wesche $(2005 ; 2010)$ developed an in-depth taxonomy of clue used by learners in lexical inferencing, which includes linguistic clue and non-linguistic clue. Linguistic clue consists of intra-lingual and inter-lingual clue. Intra-lingual clue comprises word level, sentence level, and discourse level clue. This taxonomy provides a 
comprehensive and systematic tool to classify various types of clues.

In addition to clue identification and classification, a number of studies focus on the clue use of learners with different levels of proficiency. When exploring Chinese ESL lexical inferencing, Chern (1993) observed that learners rarely used distant contextual clues and employed semantic and syntactic instead. Proficient learners tend to utilise more context clues than less proficient ones. Morrison (1996) revealed that low proficiency learners used only one type of clue, while high proficiency learners tended to use more clues; high proficiency learners excelled at using linguistic knowledge about the words, while low proficiency learners were more limited on contextual knowledge.

Based on the activation and integration of contextual and linguistic clues (bottom cues of target word itself), Haastrup (2008) proposed an eight-type continuum to describe lexical inferencing process. The advanced type of lexical inferencing is the top-ruled interactive processing with full integration of linguistic cues. Advanced learners tend to employ more advanced types of lexical inferencing. In most of these studies, however, participants were simply divided into low or high proficiency group; there are very few studies in which participants were grouped according to the stage of their language learning. Furthermore, very limited research has been conducted on lexical inferencing of Chinese EFL learners. Therefore a study on lexical inferencing of Chinese EFL learners in different language learning stages result in some insightful views on the process of lexical inferencing among Chinese EFL learners.

The research questions of this study were:

1) How do Chinese EFL learners use clue to infer the meaning of unknown word in reading?

2) What are similarities and differences of clue use in lexical inferencing among Chinese tertiary final, tertiary middle, tertiary beginning, and senior secondary year-2 EFL learners?

\section{Methodology}

\subsection{Data Elicitation Methods}

Think-aloud and stimulated recall were employed to capture the details of Chinese EFL learners' lexical inferencing. The combination of two introspection methods is widely used in research of related area. Participants were first requested to read an article, infer the meaning of target words, and at the same time to verbalize all their thoughts while inferring. This was also supplemented by the retrospection of participants on the process of lexical inferencing.

\subsection{Participants}

Participants in this study consisted of four groups of Chinese EFL learners: tertiary final participants were at the end of their tertiary education, tertiary middle were right at the middle of their tertiary education, tertiary beginning participants just began their tertiary education, and senior secondary participants were at the beginning of their second academic year. The time interval of English learning between two successive groups was two years. All tertiary participants majored their study in English language and literature. The details of participants are shown in Table 1.

Table 1. Participants of the study

\begin{tabular}{lccccc}
\hline & Introspection & Participants & Age & $\begin{array}{c}\text { Major in } \\
\text { English }\end{array}$ & Female/male \\
\hline Senior Secondary & 11 & $20.0 \%$ & $16-17$ & No & $8 / 3$ \\
Tertiary Beginning & 14 & $25.5 \%$ & $18-19$ & Yes & $11 / 3$ \\
Tertiary Middle & 15 & $27.3 \%$ & $20-21$ & Yes & $12 / 3$ \\
Tertiary Final & 15 & $27.3 \%$ & $22-24$ & Yes & $11 / 4$ \\
Total & 55 & $100 \%$ & $16-24$ & & $42 / 13$ \\
\hline
\end{tabular}

\subsection{Text and Target Words}

An 876-word article entitled Olfactory Diagnostic - Smelling Bad, selected from the science and technology section of The Economist, was used to elicit participants' lexical inferencing behaviour. Twelve target words accounted for merely $1.7 \%$ of the total number of words. All target words were content words and unknown to 
most of the learners with same level as tertiary final participants in a previous pilot test. All other words potentially unknown to senior secondary participants were covered by a glossary attached to the article.

\subsection{Procedures}

One week before individual session, all participants of introspection took part in one-hour think-aloud training session. The training consisted of introduction of think-aloud and practice on number computation, anagram, and reading comprehension.

During the think aloud sessions, each participant was presented with the article and glossary and requested to read and infer the meaning of underlined target words. Participants were also requested to verbalize what they were thinking while reading and guessing in either English or Chinese language at their will. The researcher who was the author of the paper sat behind silently except prompting participants to verbalize what they were thinking.

Immediately after reading and inferring, participants were presented the article again and requested to recall the entire process of inference for each target word. After that, participants were interviewed about the problems popping up in their guessing. The time of the process for individual participant varied from one and a half to two and a half hours. And each session was audio-recorded.

\section{Results}

The participants' think-aloud protocols and interviews were segmented and then transcribed. The subsequent analyses were based on the transcripts. The data analyses focused on identifying the clues used in inferencing. The data were analyzed qualitatively first and then followed by quantitative exploration.

\subsection{Clue Types and Taxonomy}

\subsubsection{Taxonomy of Clue}

A variety of clues were identified in transcriptions of Chinese EFL learners' protocols of lexical inferencing. Based on the taxonomy developed by Paribakht and Wesche (2005), these identified clues were subsequently classified. The depiction of each type of clue is presented in the following section, accompanied by the translation of a typical protocol sample (some may contain more than one type of clue).

\section{Linguistic Clues}

A: Intra-lingual Clues

\section{1 . Word Level}
a) Word association
b) Word collocation
c) Word morphology
d) Homonymy

2. Sentence Level

a) Sentence meaning

b) Sentence grammar

c) Punctuation

3. Discourse Level

a) Discourse meaning

b) Formal schemata

c) Text style and register

B: Inter-lingual (L1 Based) Clues

1) L1 Collocation

2) Grammar

3) Association

II. Non-linguistic Clues

World knowledge

Figure 1. The taxonomy of clue use in lexical inferencing 


\subsubsection{Linguistic Clues}

As shown in Figure 1, linguistic clues include intra-lingual and inter-lingual clues. In using intra-lingual clues, readers rely on their knowledge of the target language to infer the meaning of target word. This category includes word level, sentence level, and discourse level clues.

Word level of clues refer to features of target word, such as word morphological structure, homonymy, word association and collocation.

Word association refers to association of the target word with another familiar word or a network of words.

Example 1 Target word 'excrete'

Think-aloud (TA) ...excreted or exuded from the body crete $c r$ e te $c$ e e t e excrete should be exhale is 'respire out', should be 'respire in', this one is 'respire out' then this should be 'respire in'...

Stimulated recall (SR) ... This is 'respire out', and then this one (excrete) should be 'respire in', they two should be closely related or synonymous...

Collocation refers to the knowledge of words that frequently occur with the target word.

Example 2 Target word 'cagey'

SR I think this sentence he is cagey about details, I think it might be 'care about'. This about really remind me of care about. I put this meaning back in this sentence, and it is acceptable.

Morphological structure of word refers to the stems, prefixes and suffixes of target word.

Example 3 Target word 'immemorial'

TA Since time immemorial, this memorial, at first glance, remind me of the word memory memory memorial must be 'of memory' 'can be remembered', with the prefix of im, it is a negative one, it is an antonym to memorial, means 'not of memory' 'can't be remembered'...

Homonymy refers to mistaking the target word for another that resembles it phonologically and orthographically.

Example 4 Target word 'nuance'

SR It seems that I have come across this word before, it might mean trivial troubles. Or I might make a mistake here, I can't remember whether it is nuance or of other spelling, it seems there is an ' $\mathrm{i}$ ' in that word (nuisance)....

Sentence level clues refer to clues at sentence level, such as, the semantic meaning, the target language grammar, and punctuation rules of the sentence containing the target word.

Sentence meaning refers to the meaning of part or the entire sentence containing the target word.

Example 5 Target word 'tweak'

TA Dr Buszewski is now tweaking- tweaking his device so that it works with the biomarker profiles tweaking adjust, fine tune or improve, he is now improving his device in order to...

SR I inferred its meaning mainly from the meaning of this sentence. In order to work with the biomarker profile of a range of disease, he should improve or upgrade his device....

Sentence Grammar refers to the knowledge of the syntactic properties, the speech part, and word order constraints of the target word.

Example 6 Target word 'volatile'

TA ...to detect some vo l a t i l e to detect detect 250250 types of compound. So this volatile should be an adjective, it might mean might mean volatile volatile might mean 'complicated'....

Punctuation refers to the knowledge of rules of punctuation and their significance.

Example 7 Target word 'exude'

TA ... excreted or exuded from the body this two words exhale means 'respire out', there is a comma and or here, therefore, I think they three should share similar meaning, they might mean 'come out' from the body ...

Discourse level clues refer to the clue beyond the sentence level, which consist of discourse meaning, formal schemata, and discourse style and register. Discourse meaning refers to the perceived general meaning of the text and sentences surrounding the target word. 
Example 8 Target word 'olfactory'

TA .... ol factory ol and factory factory is gongchang/factory, olfactory diagnosis diagnosis En diagnosis is diagnosis, it should be a type of diagnosis method, olfactory, the whole article is about finding illness from the smell of breath, so it might refer to that way of diagnosis, yes, olfactory should mean that ...

Formal schemata refer to the knowledge of the macrostructure of the text, text types and discourse pattern, and organization.

Example 9 Target word 'olfactory'

SR one of the first practitioner of the field of olfactory diagnosis, olfactory it is in the title of this article, but I can't figure it out at the beginning. It must be closely related to this topic of this article. And this article is mainly about a new way of diagnosis, the smell. Therefore this word might be related to smell....

Clue of Style and register refers to the knowledge of stylistic and register variation in word choice. Apparently, the participant in following example realized that the target word is medical terminology used in restricted circumstance rather than in common daily life.

Example 10 Target word 'carcinoma'

SR She confirmed that the early stages Confirm that the early this is basal cell I think it should be this inserted phrase a type of skin cancer is an appositive to modify this word. En so it is one kind of the skin cancer, anyway it is a medical terminology, an illness....

Participants occasionally used inter-lingual clues in lexical inferencing. Such clues consist of L1 Grammar, L1 association and collocation.

L1 collocation refers to the knowledge of words that have collocation relationship with the L1 equivalent of the target word.

Example 11 Target word 'bladder'

SR I think it should be 'pernicious'. Cancer is Zhongliu/tumor, dogs can sniff out this cancer, I treated these two words as one unit with meaning of E'xing Zhongliu/ sarcomata at the very time when I translate cancer into Zhongliu/tumor, therefore bladder is E'xing/pernicious....

L1 association refers to the knowledge of words that have association relationship with the L1 equivalent of the target word.

Example 12 Target word 'olfactory'

TA .... field is land, open land, of olfactory factory is Gongchang/factory olfactory the field Gongchang/factory diagnosis diagnosis open factory diagnosis? One of the practitioners, this practitioner Oh field means domain or realm. In the domain of olfactory diagnosis, olfactory might mean collective, collective diagnosis....

SR Diagnosis, a collective diagnosis. Factory is Gongchang/factory and Gongchang is collective, therefore olfactory means collective....

L1 Grammar refers to the L1 knowledge of the syntactic properties, the speech part, and word order constraints of $\mathrm{L} 1$ equivalent word of the target word.

Example 13 Target word 'volatile'

TA ...vola volatile organic compounds in the air exhaled exhaled from the lungs, 250 organic This should be a should be a unit word 250 vala volatile organic compound in the air exhaled from lungs...

SR There is a number ahead of this word. In most cases numbers are only followed by nouns and unit words. Since there is already a noun, it must be a unit word...

Unit word is a unique part of speech in Chinese language, which lies after the quantity number that modifies a noun. There is no equivalent speech part in English language.

\subsubsection{Non-Linguistic Clues}

Non-linguistic clues refer to general background knowledge of the topic or world knowledge employed during inferring.

Example 14 Target word 'bladder' and 'prostate' 
TA.... This bladder and this prostate cancer, What the dog can detect must be something coming out of human's breath, therefore it must be related to internal organs, bladder prostate internal organs, lung is lung, liver is liver, they are not stomach, stomach is stomach, what else internal organs are there ...

\subsection{Clue Use Patterns in Lexical Inferencing}

Data were analysed in a quantitative manner as well. Frequencies of clue use were calculated using simple count of the number of times the clue was used by each participant. The result is presented in Table 2. A variety of clues, 14 different sub-types, were used by Chinese EFL learners in their lexical inferencing. Sentence meaning was by far the most important clue used by all participants; this accounted for $43.58 \%$ of total clue use. Sentence meaning was likely to be important because lexical inferencing is meaning-related, and sentence meaning is the neighbouring information resource of the target word. Discourse meaning ranked third, accounting for $14.73 \%$ of total clue use. Sentence meaning and discourse meaning collectively accounted for $54.8 \%$ of total clue use. Morphology was next to sentence meaning in importance, which accounted for $28.76 \%$ of total clue use. After the three major clues, sentence grammar, world knowledge, word association, homonymy, and L1 grammar played a relatively restricted role, accounting for $6.13 \%, 3.48 \%, 1.81 \%, 1.81 \%$, and $1.16 \%$, respectively. Clues such as formal schema, punctuation, L1 collocation, word collocation, text style and register, and L1 association played very restricted roles in lexical inferencing; these accounted for merely $0.52 \%, 0.45 \%, 0.45 \%, 0.32 \%$, $0.19 \%$, and $0.13 \%$, respectively. On average, 2.4 clues were used for each inference.

A number of patterns of clue use were shared by all groups. Firstly, a wide variety of clues were used by each group; seven to eight of 14 different sub-types of clues were reported as accounting for at least $1 \%$ of total clue use of the group. Secondly, the importance of sentence meaning, morphology, and discourse meaning clue use were shown across groups. Sentence meaning accounted for clue use ranging from $41.07 \%$ to $48.35 \%$; morphology accounted for $20.15 \%$ to $31.02 \%$; and discourse meaning accounted for $8.79 \%$ to $14.73 \%$ of clue use.

Certain patterns that could distinguish the four groups were observed as well. Firstly, a wide variety of clues were used by each group; 7 to 8 of 14 different sub-types of clue were reported as accounting for at least $1 \%$ total clue use of the group. Secondly, the importance of sentence meaning, morphology, and discourse meaning clue use were shown across groups. Sentence meaning accounted for clue use ranging from 41.07 to $48.35 \%$; morphology accounted for 20.15 to $31.02 \%$; and discourse meaning accounts for $8.79-14.73 \%$ of clue use.

There were also some patterns that could distinguish the four groups. Firstly, senior secondary participants, compared with other participants, employed more sentence meaning but less morphology clue. Then, seemingly there was a slight increasing tendency of word association clue use from senior secondary to tertiary final group, a slight increasing tendency of discourse meaning clue from tertiary beginning to tertiary final group, and a slight decreasing tendency of sentence grammar clue from senior secondary to tertiary final group.

Table 3 presents the ranking of each clue. All sub-type clues were ranked in terms of percentage of their reported use in each group, showing the overall comparative overall importance of the various clues that participants use in lexical inferencing. One of the merits of this table is that it visually classifies the clues used into different frequency zones. It also presents percentage (close to the nearest integer, or decimal in 'rare' case) to cast light on the main tendencies.

As demonstrated in Table 3, no significant difference in sentence meaning and word morphology among groups were observed according to ranking order. However, unlike that of other groups, word morphology of senior secondary group was 'frequent' not 'very frequent'. In terms of discourse meaning, the ranking was 'occasional' in senior secondary and tertiary beginning group, but 'frequent' in tertiary middle and final group. As to sentence grammar, the ranking was 'frequent' in senior secondary group while 'occasional' in other groups. As to the ranking of word association, it is 'rare for senior secondary group, rather than 'occasional' as that of other groups. For tertiary beginning group, it was rather backward in the zone of 'occasional' compared with that of other two tertiary groups. As to the ranking of L1 grammar, it was 'occasional' in all groups except the tertiary beginning group. In tertiary beginning group, it was 'rare'. It seems that tertiary beginning participants used less L1 grammar clue in lexical inferencing.

There were also some discrepancies within zones, especially in 'occasional' zone. As shown in Table 7, the ranking of world knowledge, though, was 'occasional' across group; there was still a slight difference. It ranked fifth in all other groups except tertiary middle. The ranking of homonymy was rather backward in 'occasional' zone in senior secondary, tertiary beginning, and tertiary final group (ranked the sixth or seventh), but relatively forward in tertiary middle (ranked the fifth). 
Table 2. Detailed clue use in lexical inferencing (Count/Percentage)

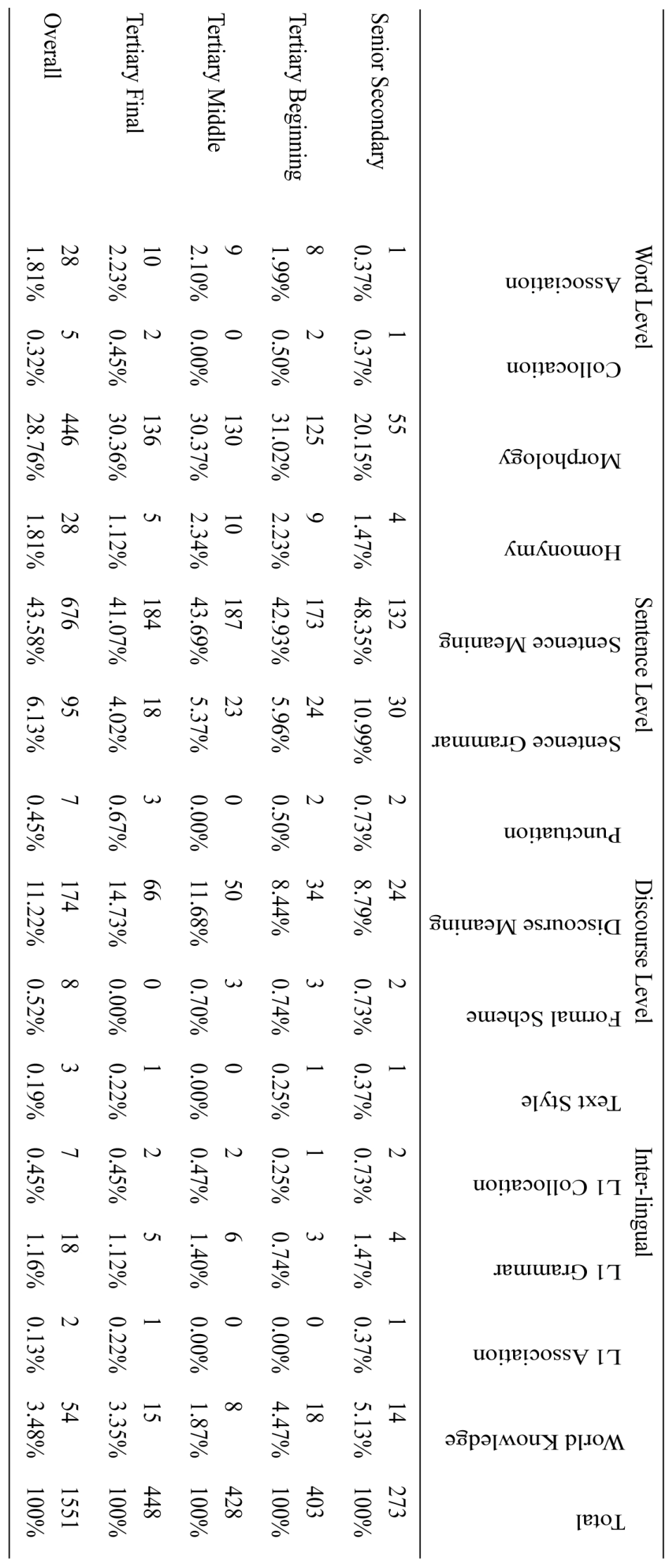


Table 3. Ranking of clues used in lexical inferencing within relative frequency zones

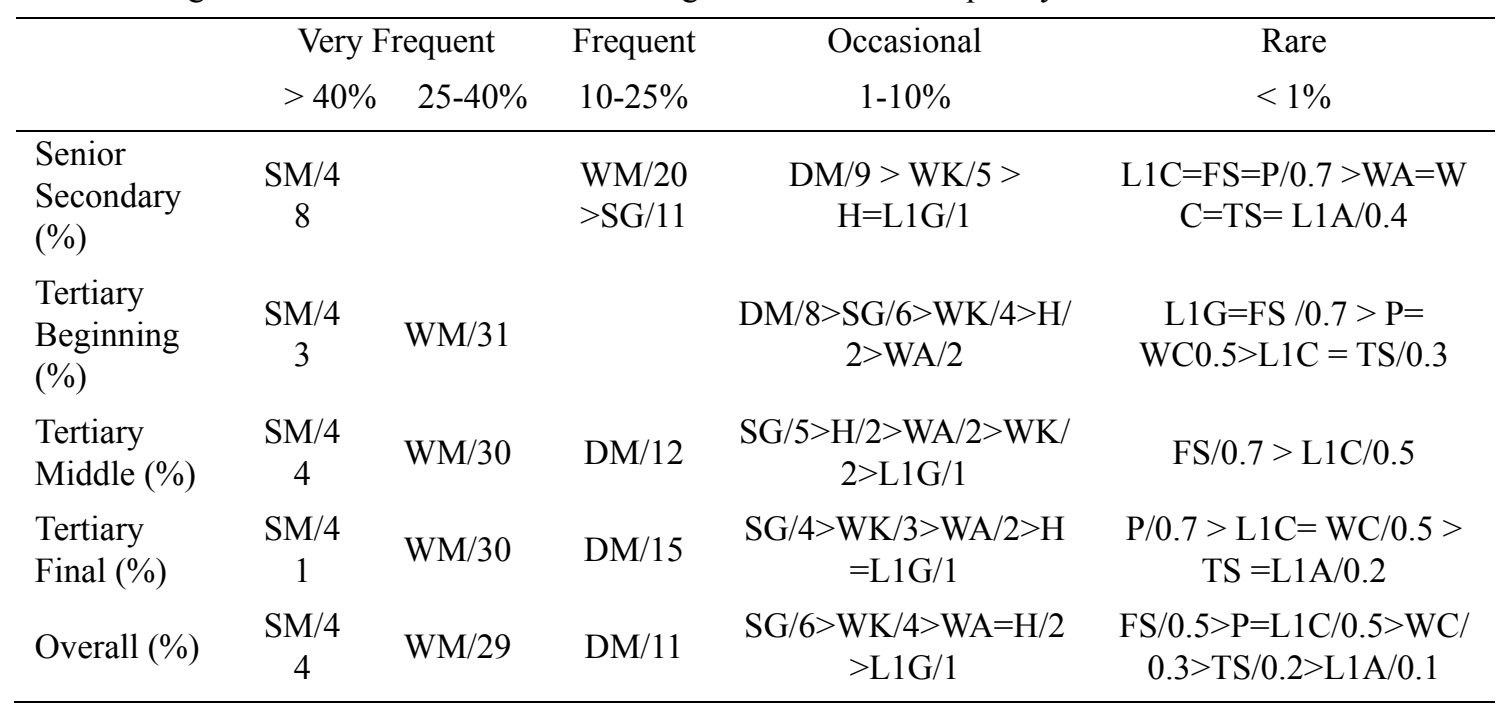

Note: Percentages are closed to the nearest integer or decimal (in cases of 'rare').

$\mathrm{SM}=$ Sentence Meaning; WM=Word Morphology; WC=Word Collocation; WA=Word Association; $\mathrm{H}=$ Homonymy; $\mathrm{SG}=$ Sentence Grammar; $\mathrm{DM}=$ Discourse Meaning; $\mathrm{WK}=$ World Knowledge; $\mathrm{L} 1 \mathrm{G}=\mathrm{L} 1$ Grammar; L1C=L1 Collocation; L1A=L1 Association; FS=Formal Schemata; TS=Text Style \& Register; P=Punctuation

Taking into account of the low frequency, the ranking of the clues within 'rare' zone was susceptible to accidental influence. It is difficult to claim any tendencies of clues use from their ranking in this zone.

\subsection{Multiple or Single Clue Use in Lexical Inferencing}

Another aspect of clue use in lexical inferencing is the number of clue used in each inference. Participants in four groups used multiple clues to generate the meaning of some target words, and single clue for others. Overall, as demonstrated by Table 4, $28.9 \%$ of total inferences were single clue inference and $71.1 \%$ were multiple clue inferences. The single clue inference accounted for $40.9 \%$ in senior secondary group, $29.4 \%$ in tertiary beginning group, $25 \%$ in tertiary middle group, and $23.3 \%$ in tertiary final group. It seems that there was a decreasing tendency of single clue inference from senior secondary group to tertiary final group. As to multiple clue inference, on the contrary, it was observed that there were tendencies of increasing from $59.1 \%$ for the senior secondary group to $70.6 \%$ for the tertiary beginning group, and $75.0 \%$ for the tertiary middle group to $76.7 \%$ for the tertiary final group.

Table 4. Multiple clue use across group (inference)

\begin{tabular}{lllll}
\hline & Single Clue Use & $\begin{array}{l}\text { Multiple } \\
\text { Clue Use }\end{array}$ & Total & Clues/Inference \\
\hline Senior Secondary & 54 & 78 & 132 & 1.9 \\
& $40.9 \%$ & $59.1 \%$ & $100 \%$ & \\
Tertiary Beginning & 53 & 127 & 180 & 2.4 \\
& $29.4 \%$ & $70.6 \%$ & $100 \%$ & \\
Tertiary Middle & 45 & 135 & 180 & 2.4 \\
& $25.0 \%$ & $75.0 \%$ & $100 \%$ & \\
Tertiary Final & 42 & 138 & 180 & 2.5 \\
& $23.3 \%$ & $76.7 \%$ & $100 \%$ & \\
Overall & 194 & 478 & 672 & 2.4 \\
& $28.9 \%$ & $71.1 \%$ & $100 \%$ & \\
\hline
\end{tabular}


The Pearson Chi-Square results demonstrated that the difference across groups was significant at $p$ level of 0.01 .

Table 5. Pearson Chi-Square result

\begin{tabular}{llll}
\hline & Value & df & Asymp. Sig. (2-sided) \\
\hline Pearson Chi-Square & $15.055(\mathrm{a})$ & 3 & .002 \\
Likelihood Ratio & 14.222 & 3 & .003 \\
Linear-by-Linear Association & 9.179 & 1 & .002 \\
N of Valid Cases & 659 & &
\end{tabular}

a 0 cells $(.0 \%)$ have expected count less than 5 . The minimum expected count is 36.25 .

\section{Discussion and Implications}

The results demonstrate that in lexical inferencing Chinese EFL participants employed a number of clues varied from word and sentence level to discourse level, from target language to first language, from linguistic to non-linguistic background knowledge. The findings reveal that prior linguistic and non-linguistic knowledge interacts with contextual clue in the process of Chinese EFL learners' lexical inferencing.

Many findings of this study are consistent with that of previous studies (Bengeleil \& Paribakht, 2004; Paribakht, 2005; Paribakht \& Wesche, 1999; Wesche \& Paribakht, 2010). For instance, the clue types identified in this study are similar to those in previous research; sentence meaning, word morphology and discourse meaning are three most frequently used clues. In contrast to findings from previous studies, it seems that more word morphology clues were used by Chinese EFL learners and L1 grammar clue was identified as a new type kind of clue. In this study, the clue of word morphology ranked second, just after sentence meaning but it ranked third or lower in previous studies (Bengeleil \& Paribakht, 2004; Paribakht \& Wesche, 1999). This pattern might be a result of the influence of the Chinese language. Majority of Chinese characters consist of two radicals, one indicates its sound while the other indicates its meaning. Therefore majority of unknown characters in reading can be settled by analyzing their structures. As to the new finding of L1 grammar clue use in Chinese EFL lexical inferencing, L1 grammar as a clue appears to be involved in L2 lexical inferencing at all times, but the linguistic similarities between L1 and L2 studied in previous research may not allow researchers to identify the role of L1 grammar clue in lexical inferencing. The uniqueness of Chinese language grammar and contrastive differences between Chinese and English grammar in EFL learners may shed light on the unique influence of L1 grammar as a clue.

The findings further reveal that the four groups of Chinese EFL learners, despite their diverse stages of English learning, share several patterns of clue use in lexical inferencing. All groups used similar types of clues. Among these types of clues, sentence meaning, word morphology and discourse meaning were three most frequently used. However, there were also some discrepancies across groups. The less word morphology used in the senior secondary group might be caused by the fact that they were not as competent as other participants in morphological knowledge. Their introspection protocols seemed to confirm this. The discrepancy in discourse clue use across groups might also be caused by the fact that competence in text processing of senior secondary and tertiary beginning participants was not as high as that of tertiary middle and final participants. Therefore some potential discourse clues were beyond their access. As to the discrepancy of homonymy, protocol Example 4 might provide some extra information to understand this situation. This tertiary middle participant mistook the target 'nuance' as 'nuisance'. For senior secondary and tertiary beginning participants, there was little chance to encounter the word 'nuisance'; by contrast, tertiary middle or final participants appeared to be more likely to know this word and more likely to commit this mistake.

Some pedagogical implications can be drawn from this study. First, for senior secondary learners, the apparent deficiency of morphological knowledge suggests the inclusion of morphological knowledge in secondary English instruction. Learners should be familiar with majority of the common prefixes and suffixes. Secondly, introspective protocols show that many learners could not identify some contextual clues; and interviews indicated that they had no formal training and practice on lexical inferencing. Formal training and practice may enhance their lexical inferencing performance. Studies (Fraser, 1997) have shown that improved awareness of all types of potential clues within or outside text may assist learners to enhance their efficiency and effectiveness in lexical inferencing. 


\section{References}

Ames, W. S. (1966). The development of a classification scheme of contextual aids. Reading Research Quarterly, 2, 57-82.

Bengeleil, N. F., \& Paribakht, S. (2004). L2 Reading Proficiency and Lexical Inferencing by University EFL Learners. Canadian Modern Language Review, 61(2), 225-249. http://dx.doi.org/10.3138/cmlr.61.2.225

Carton, A. S. (1971). Inferencing: A process in using and learning language. In P. Pimsleur, \& T. Quinn (Eds.), The psychology of second language learning (pp. 45-58). Cambridge: Cambridge University Press.

Chern, C. L. (1993). Chinese students' word-solving strategies in reading in English. In T. Huckin, M. Haynes, \& J. Coady (Eds.), Second language reading and vocabulary learning (pp. 67-85). Norwood, NJ: Ablex Publishing Corporation.

De Bot, K., Paribakht, S., \& Wesche, M. (1997). Towards a lexical processing model for the study of second language vocabulary acquisition: Evidence from ESL reading. Studies in Second Language Acquisition, 19(2), 309-329. Retrieved from http://journals.cambridge.org/article_S0272263197003021

Fraser, C. A. (1999). Lexical processing strategy use and vocabulary learning through reading. Studies in Second Language Acquisition, 21, 225-241. Retrieved from http://journals.cambridge.org/article_S0272263199002041

Haastrup, K. (1991). Lexical inferencing procedures or talking about words. Tübingen, Germany: Gunter Narr.

Haastrup, K. (2008). Lexical Inferencing Procedures in Two Languages. In D. Albrechtsen, K. Haastrup, \& B. Henriksen (Eds.), Vocabulary and Writing in First and Second Language: Process and Development. Basingstoke, Hampshire: Palgrave Macmillan.

Herman, P. A., Anderson, R. C., Pearson, P., \& Nagy, W. E. (1987). Incidental acquisition of word meaning from expositions with varied text features. Reading Research Quarterly, 22(3), 263-284.

Morrison, L. (1996). Talking about words: A study of French as a second language leaerners' lexical inferencing procedures. Canadian Modern language journal, 53, 41-75.

Paribakht, S. (2005). The Influence of First Language Lexicalization on Second Language Lexical Inferencing: A Study of Farsi-Speaking Learners of English as a Foreign Language. Language Learning, 55(4), 701-748. http://dx.doi.org/10.1111/j.0023-8333.2005.00321.x

Paribakht, S., \& Wesche, M. (1999). Reading and incidental L2 vocabulary acquisition: An introspective study of lexical inferencing. Studies in Second Language Acquisition, 21, 195-224. Retrieved form http://journals.cambridge.org/article_S027226319900203X

Sternberg, R. J. (1987). Most Vocabulary is learned from context. In M. G. McKeown, \& M. E. Curtis (Eds.), The nature of vocabulary acquisiton (pp. 89-105). Hillsdale, N. J.: Erlbaum.

Wesche, M. B., \& Paribakht, T. S. (2010). Lexical Inferencing in a First and Second Language: Cross-linguistic Dimensions. Bristol: Multilingual Matters.

\section{Copyrights}

Copyright for this article is retained by the author(s), with first publication rights granted to the journal.

This is an open-access article distributed under the terms and conditions of the Creative Commons Attribution license (http://creativecommons.org/licenses/by/3.0/). 